04

\title{
Особенности формирования рентгеновских потоков волноводами-резонаторами различных конструкций
}

\author{
(C) B.K. Егоров ${ }^{1}$, E.B. Егоров ${ }^{1,2}$ \\ ${ }^{1}$ Институт проблем технологии микроэлектроники и особочистых материалов РАН, \\ 142432 Черноголовка, Московская обл., Россия \\ 2 Российский университет дружбы народов, \\ 117198 Москва, Россия \\ e-mail: egorov@iptm.ru
}

Поступила в редакцию 12.11.2017 г.

\begin{abstract}
Кратко описаны явление полного внешнего отражения (ПВО) потоков квазимонохроматического рентгеновского излучения на материальном интерфейсе, эффект волноводно-резонансного распространения этих потоков в наноразмерных протяженных щелевых зазорах, а также устройство, функционирующее на базе этого эффекта: плоский рентгеновский волновод-резонатор (ПРВР). Представлены экспериментальные данные по формированию потока рентгеновских лучей составным плоским волноводом-резонатором (СПРВР) и предложена модель, описывающая снижение угловой расходимости формируемого потока без уменьшения интегральной интенсивности. Модель основана на концепции частичного углового туннелирования радиационного потока в промежутке между двумя последовательно установленными и взаимно съюстированными волноводами-резонаторами, которое реализуется вследствие взаимодействия интерференционных полей стоячих рентгеновских волн, возбужденных излучением, транспортируемым щелевыми зазорами этих волноводов-резонаторов.
\end{abstract}

DOI: $10.21883 /$ OS.2018.06.46085.262-17

\section{Введение}

Важным аспектом любой спектрометрии является разработка эффективных процедур формирования выходных потоков ожидаемых спектрометрических продуктов. Разработка таких процедур, как правило, опирается на наличие реальных физических явлений, позволяющих варьировать характеристики этих потоков. Энергия и геометрические параметры пучков заряженных частиц могут легко изменяться варьированием значений и пространственных распределений электрических и магнитных полей. Для управления потоками оптического излучения могут применяться методы, построенные на явлениях зеркального и полного внутреннего отражения [1], а также включающие эффекты частичного пропускания и нарушенного полного внутреннего отражения [2-4]. Имеется большое количество оптических элементов и устройств, использующих явление рефракции (преломления) потоков светового излучения [5]. Кроме того, для управления параметрами световых потоков на практике применяется множество устройств, функционирование которых основано на использовании целого ряда поляризационных явлений, таких как эффекты Брюстера, Керра, Фарадея и др. [6]. В то же время возможности управления параметрами потоков рентгеновского излучения, особенно в жесткой энергетической области, оказываются весьма ограниченными.

Исследование взаимодействия рентгеновского излучения с материалами показали, что имеются два фундаментальных явления, позволяющие изменять геометрические и энергетические параметры распространения потоков данного излучения. Это явление брегговской дифракции [7] и полное внешнее отражение на материальных интерфейсах [8]. Важными дополнениями к ним являются эффект Бормана, иногда именуемый эффектом аномального прохождения потоков рентгеновских лучей через совершенные монокристаллы [9], и едва наблюдаемый эффект рефракции [10]. В самое последнее время появились устройства, функционирующие на основе волноводно-резонансного распространения рентгеновских потоков - плоские рентгеновские волноводырезонаторы (ПРВР) [11]. На основании особенностей формирования потоков рентгеновского излучения этими устройствами оказалось возможным разработать еще один специфический инструмент, позволяющий влиять на параметры формируемого потока - составной плоский рентгеновский волновод-резонатор [12]. В настоящей работе предпринята попытка построения модели функционирования этого оригинального устройства.

\section{Плоские рентгеновские волноводы-резонаторы и особенности их функционирования}

Экспериментальные и теоретические факторы, позволившие выявить существование волноводно-резонансного механизма распространения потоков квазимонохроматического рентгеновского излучения, в первую очередь оказались связанными с наличием высокой рентгено-транспортной эффективности так называемых 
„бесщелевых“ рентгеновских коллиматоров [13-15]. К сожалению, первоначальная интерпретация полученных в этих работах экспериментальных данных, как и последующие их обсуждения [16,17], основывались на широко известном механизме распространения рентгеновского излучения - многократном полном внешнем отражении. Этот механизм является базовым для описания функционирования устройств рентгеновской моно- и поликапиллярной оптики, первый вариант которых был предложен еще в 1951 г. [18]. Кардинальное влияние на переосмысливание экспериментальных данных по рентгено-транспортным свойствам „бесщелевых“ рентгеновских коллиматоров и высокой эффективности их применения для спектрометрии РФА ПВО [19-21] оказала работа Бедзика с сотрудниками [22], в которой было экспериментально показано появление интерференционного поля стоячей рентгеновской волны (ИПСРВ) в условиях ПВО потока квазимонохроматического рентгеновского излучения на плоском материальном интерфейсе. На рис. 1 представлена модель, наглядно представляющая это явление. Квазимонохроматичность излучения характеризуется наличием среднего значения длины волны $\lambda_{0}$, степенью монохроматичности $\Delta \lambda$ и параметром длины когерентности $L=\lambda_{0}^{2} / \Delta \lambda$, который фактически отражает продольный размер рентгеновского фотона [23]. Поперечный размер фотона в значительной степени определяется условиями эксперимента [24-26], а при реализации ПВО рентгеновского излучения продольный и поперечный размеры фотонов оказываются приблизительно совпадающими [27]. Период стоячей рентгеновской волны, возбуждаемой в условиях ПВО над материальным интерфейсом, определяется [23] выражением

$$
D=\frac{\lambda_{0}}{2 \sin \theta} \approx \frac{\lambda_{0}}{2 \theta} .
$$

Вследствие малости углов ПВО (критический угол $\theta_{c}$ обычно составляет $0.1-0.2^{\circ}$ ) замена $\sin \theta$ на $\theta$ в выражении (1) представляется допустимой. Аналитические значения критических углов ПВО могут быть оценены на основании различных подходов к описанию взаимодействия потоков рентгеновского излучения с материалом. Наиболее часто для этих целей используется френелевский подход [28,29], в соответствии с которым материал описывается путем введения диэлектрической проницаемости $\varepsilon$ и показателя преломления $n{ }^{1}$

$$
n=\sqrt{\varepsilon}=1-\delta-i \beta,
$$

где параметр $\delta$ представляется фактором поляризации среды, а $\beta-$ величиной, отвечающей за поглощение рентгеновского излучения материалом. Используя этот

\footnotetext{
${ }^{1}$ Важно отметить, что подобный подход является принципиально незаконным, поскольку длина волны рентгеновского излучения меньше межатомного расстояния и параметр $\varepsilon$ должен рассматриваться зависящим от координаты [30], а введение коэффициента преломления теряет физический смысл.
}

подход, аналитическое выражение для значения критического угла ПВО представляется [29] в форме

$$
\theta_{c}=\sqrt{2 \delta}=\sqrt{8.24 \cdot 10^{4} \frac{Z}{A} \frac{\rho}{E^{2}}},
$$

где $Z, A$ и $\rho$ являются характеристиками материала: $Z$ представляет собой усредненный заряд атомов, составляющих рефлекторный материал, $A-$ усредненный атомный вес, $\rho$ - плотность. Величина $E$ отражает энергию квантов рентгеновского излучения в $\mathrm{keV}$.

В альтернативном подходе для описания взаимодействия потока рентгеновских лучей с материальными структурами и представления аналитического выражения критического угла ПВО вводится параметр потенциала [31]. Под потенциалом вакуума при этом предполагается постоянная величина $V_{1}$, а для материала вводится среднее значение потенциала $V_{2}$. Данный подход, помимо введения среднего значения потенциала, подразумевает наличие зависимости потенциальной функции материала от координаты и введения переходной области в районе интерфейса материал/вакуум по аналогии дополнения френелевской модели введением в рассмотрение переходного слоя при интерпретации эффекта Брюстера [32]. В соответствии с использованием потенциального подхода значение критического угла ПВО определяется [31] выражением

$$
\theta_{c}=\arcsin \sqrt{\frac{E-V_{2}}{E-V_{1}}}=\arcsin \sqrt{1-\frac{V_{2}-V_{1}}{E-V_{1}}},
$$

где $E-$ энергия квантов рентгеновского излучения. Данное выражение может быть значительно упрощено, если принять потенциал вакуума за начало отсчета и учесть, что экспериментально полученные значения критических углов ПВО различных материалов весьма малы [33]. ${ }^{2}$ В то же время необходимо признать, что потенциальный подход является в настоящее время экзотическим не только для описания ПВО, но и в целом для трактования взаимодействия потока рентгеновских лучей с материалом. Общепринятым, хотя и незаконно, считается френелевский подход [22,28,29,33].

При падении потока рентгеновских лучей на материальный интерфейс независимо от угла его падения какая-то часть потока отражается, а оставшаяся часть проходит в объем материала. В соответствии с рис. 1, моделирующим явление ПВО, падающему, отраженному и прошедшему в глубь материала потокам могут быть приписаны волновые векторы $\mathbf{k}, \mathbf{k}_{R}$ и $\mathbf{k}_{T}$ соответственно. Если принять, что эти векторы лежат в плоскости $x-z$, причем ось $z$ соответствует нормали к поверхности,

\footnotetext{
2 Следует иметь в виду, что данная концепция имеет свои недостатки, поскольку экспериментально установлено, что значения критических углов ПВО для разных ориентаций монокристаллов, в частности для $\mathrm{Si}$, хотя и незначительно, но различаются.
} 


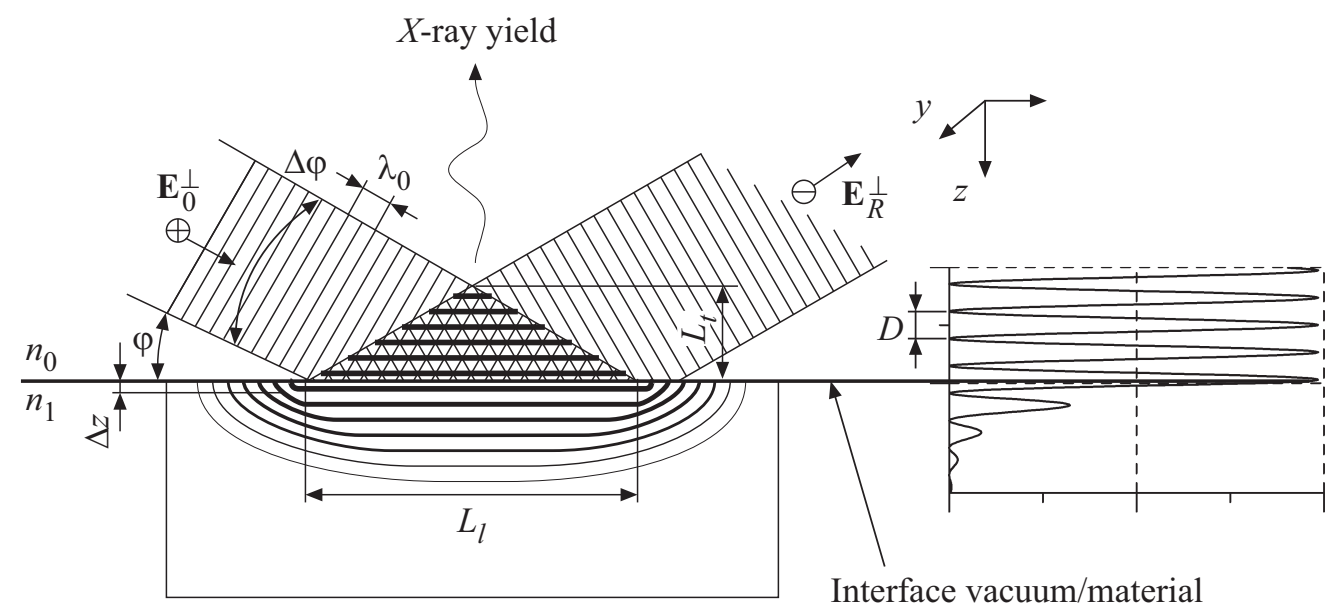

Рис. 1. Упрощенная геометрическая модель ПВО потока квазимонохроматического рентгеновского излучения на материальном интерфейсе [26]. Рассматривается поток, поляризованный перпендикулярно плоскости падения. $L_{l}$ и $L_{t}-$ продольный и поперечный размеры области интерференционного поля стоячей рентгеновской волны, $D-$ период этого поля, $\Delta z-$ глубина проникновения [8].

то электрические поля падающей, отраженной и прошедшей через интерфейс бегущих волн могут быть описаны [22,33] выражениями

$$
\begin{aligned}
& \mathbf{e}_{0}(\mathbf{r}, t)=\mathbf{E}_{0}^{\perp} \exp i\left[\omega t-2 \pi\left(k_{0 x} x-k_{0 z} z\right)\right], \\
& \mathbf{e}_{R}(\mathbf{r}, t)=\mathbf{E}_{R}^{\perp} \exp i\left[\omega t-2 \pi\left(k_{1 x} x-k_{1 z} z\right)\right], \\
& \mathbf{e}_{T}(\mathbf{r}, t)=\mathbf{E}_{T}^{\perp} \exp i\left[\omega t-2 \pi\left(k_{T x} x-k_{T z} z\right)\right] .
\end{aligned}
$$

Для удобства рассмотрения координата интерфейса по оси $z$ будет соответствовать нулю и будет рассматриваться только $\sigma$-поляризация, т. е. векторы $\mathbf{E}_{0}^{\perp}, \mathbf{E}_{R}^{\perp}$ и $\mathbf{E}_{T}^{\perp}$ перпендикулярные плоскости $x-z$. В соответствии с френелевским подходом [22,33] комплексные коэффициенты амплитуд отражения и преломления имеют вид

$$
\begin{aligned}
& \frac{E_{R}^{\perp}}{E_{0}^{\perp}}=\left|\frac{\mathbf{E}_{R}^{\perp}}{\mathbf{E}_{0}^{\perp}}\right| e^{i r}=\frac{\theta-\sqrt{\theta^{2}-2 \delta-2 i \beta}}{\theta-\sqrt{\theta^{2}+2 \delta-2 i \beta}} e^{i r}, \\
& \frac{E_{T}^{\perp}}{E_{0}^{\perp}}=\left|\frac{\mathbf{E}_{T}^{\perp}}{\mathbf{E}_{0}^{\perp}}\right| e^{i r}=\frac{2 \sqrt{\theta^{2}-2 \delta-2 i \beta}}{\theta-\sqrt{\theta^{2}+2 \delta-2 i \beta}} e^{i r} .
\end{aligned}
$$

Вслед за авторами работ [28,29], которые ввели упрощающее обозначение

$$
\sqrt{\theta-2 \delta-2 i \beta}=a+i b,
$$

где параметры $a$ и $b$ имеют вид

$$
\begin{aligned}
& a^{2}=1 / 2\left(\sqrt{\left(\theta^{2}-2 \delta\right)^{2}+4 \beta^{2}}+\left(\theta^{2}-2 \delta\right)\right), \\
& b^{2}=1 / 2\left(\sqrt{\left(\theta^{2}-2 \delta\right)^{2}+4 \beta^{2}}-\left(\theta^{2}-2 \delta\right)\right),
\end{aligned}
$$

выражения (6) могут быть преобразованы следующим образом:

$$
\begin{aligned}
& \frac{\mathbf{E}_{R}^{\perp}}{\mathbf{E}_{0}^{\perp}}=\frac{(\theta-a)-b i}{(\theta+a)+b i}, \\
& \frac{\mathbf{E}_{T}^{\perp}}{\mathbf{E}_{0}^{\perp}}=\frac{2(a+b i)}{(\theta+a)+b i} .
\end{aligned}
$$

Используя фактор малости углов ПВО и процедуру преобразования, выполненную в работе [33], можно представить выражение для электрического поля, прошедшего через интерфейс потока, в следующей форме:

$$
\begin{aligned}
& \mathbf{e}(x, z, t)=\left|\mathbf{E}_{0}^{\perp}\right| \frac{2(a+i b)}{(\theta+a)+i b} \\
& \quad \times \exp \left(-\frac{2 \pi i}{\lambda}(a+b i)\right) \exp \left(i\left(\omega t-k_{x} x\right)\right) .
\end{aligned}
$$

Это выражение показывает, что вдоль оси $z$ поле потенциально убывает, но при этом распространяется вдоль оси $x$. Следовательно, нужно ожидать, что фотон рентгеновского излучения, упавший на интерфейс вакуум/материал под углом ПВО, будет эмитирован материалом на некотором расстоянии от места его падения. Подобное смещение в литературе принято называть сдвигом Гуса-Хансен [34,35]. Его величина описывается [36] выражением

$$
d=\frac{\lambda}{\pi} \frac{1}{\sqrt{n_{0}^{2} \cos ^{2} \theta-n_{1}^{2}}},
$$

где $n_{0}$ - показатель преломления для вакуума, а $n_{1}-$ для материала рефлектора. Поскольку отражение является полным, не зависящая от времени функциональная часть выражения (10) будет описывать стационарное 
распределение электрического поля в объеме материала рефлектора, а интенсивность отраженного потока будет определяться первым из соотношений (9). При отсутствии поглощения $\left|\mathbf{E}_{R}^{\perp}\right|$ будет совпадать с $\left|\mathbf{E}_{0}^{\perp}\right|$. Для углов ПВО, меньших критического, сдвиг ГусаХансен не превышает длины когерентности рентгеновского излучения (например, сдвиг Гуса-Хансен для угла падения потока излучения $\mathrm{Cu} K_{\alpha}$ на кварцевый рефлектор $\theta \approx 0^{\circ}$ равен $28 \mathrm{~nm}$, в то время как длина когерентности этого излучения $L=\lambda_{0}^{2} / \Delta \lambda=511 \mathrm{~nm}$ ). Поэтому мы вправе ожидать появления области интерференции, возникающей в результате взаимодействия падающего и отраженного рентгеновских потоков, впервые экспериментально наблюдавшегося Бедзиком [22]. При наличии двух плоских параллельных рефлекторов, расположенных на некотором расстоянии $s$, попадающий в этот зазор поток квазимонохроматического рентгеновского излучения испытывает последовательное многократное ПВО с образованием набора локальных областей интерференционного поля стоячей рентгеновской волны (рис. 2,a). Если зазор между рефлекторами достаточно широк, локальные интерференционные области не пересекаются. Однако при ширине зазора, меньшей половины длины когерентности излучения, транспортируемого щелевым зазором, происходит качественное изменение распределения электрического поля потока рентгеновского излучения в зазоре с образованием однородного интерференционного поля стоячей рентгеновской волны (рис. 2, $b$ ). Явление, связанное с образованием однородного интерференционного поля, было зарегистрировано экспериментально и послужило основанием для предположения наличия специфического механизма распространения потоков квазимонохроматического рентгеновского излучения, характерного для узких планарных протяженных щелевых зазоров, образованных плоскими полированными диэлектрическими рефлекторами [37]. В стационарных условиях основная интенсивность интерференционного поля оказывается сосредоточенной в воздушном (вакуумном) щелевом зазоре, но некоторая его часть оказывается связанной с объемами рефлекторов (рис. 3). По этой причине механизм волноводно-резонансного распространения рентгеновского излучения характеризуется некоторым ослаблением потока, обусловленным коэффициентом поглощения излучения материалом рефлекторов $\mu$. Интенсивность на выходе такой щелевой структуры, названной ПРВР, определяется [37] выражением

$$
J(\theta, l)=J_{0} e^{-\alpha \mu l},
$$

где параметр $\alpha$ является функцией длины волны транспортируемого излучения, угла захвата рентгеновского потока, ширины щелевого зазора и плотности материала рефлектора, $l$ - длина щелевого зазора. Параметр $\alpha$ достаточно мал, поскольку экспериментальные исследования показали, что при длине зазоров в несколько десятков сантиметров наблюдается ослабление потока лишь на несколько процентов.

\section{Свойства и особенности ПРВР различных конструкций}

Конструкция ПРВР определяется условиями возникновения и поддержания в стационарном режиме однородного интерференционного поля стоячей рентгеновской волны в щелевом пространстве, транспортирующем поток квазимонохроматического рентгеновского излучения. Согласно выполненным оценкам, такое поле реализуется в плоском протяженном щелевом зазоре, образованном плоскими полированными диэлектрическими рефлекторами, если расстояние между ними не выходит за рамки следующего интервала [37]:

$$
\sqrt{\frac{\pi m c^{2} A}{2 e^{2} N Z^{\prime} \rho}} \leq s \leq \frac{\lambda^{2}}{2 \Delta \lambda}=L / 2,
$$

где $e$ и $m$ являются зарядом и массой электрона, $c$ - скорость света в вакууме, $N$ - число Авогадро, $Z^{\prime}$ и $A$ - эффективный заряд ядра и атомная масса атомов материала рефлекторов, $\rho$ - плотность этого материала, $\lambda_{0}$ и $\Delta \lambda-$ средняя длина волны и степень монохроматичности транспортируемого излучения. Верхний предел ширины щелевого зазора в соответствии с выражением (13) определяется половиной параметра длины когерентности $L$ транспортируемого излучения. Нижний предел определяется тем, что период стоячей волны, возникающей в протяженном щелевом зазоре, не может превышать ширину этого зазора. При возникновении такого превышения резко возрастает поглощение транспортируемого потока и, следовательно, искажаются условия его волноводно-резонансного распространения. Таким образом, рассматриваемая модель предполагает, что ПРВР не может транспортировать рентгеновский поток, падающий на его входной срез под нулевым углом. Волновод-резонатор может транспортировать малорасходящиеся рентгеновские пучки, если они падают на входной срез под углами, рассчитанными в соответствии с выражением (13).

Другим важным фактором, характеризующим ПРВР и следующим из выражения (13), является наноразмерность ширины формируемого им радиационного потока. Как было указано выше, длина когерентности характеристических излучений, генерируемых лабораторными рентгеновскими источниками, соответствует наноразмерному диапазону.

Не менее существенным параметром ПРВР, подробно охарактеризованном в предыдущем разделе, является его высокая транспортная эффективность. Экспериментальные исследования степени ослабления распространяющегося в его щелевом зазоре рентгеновского потока показали, что при высоком качестве полировки рабочих 


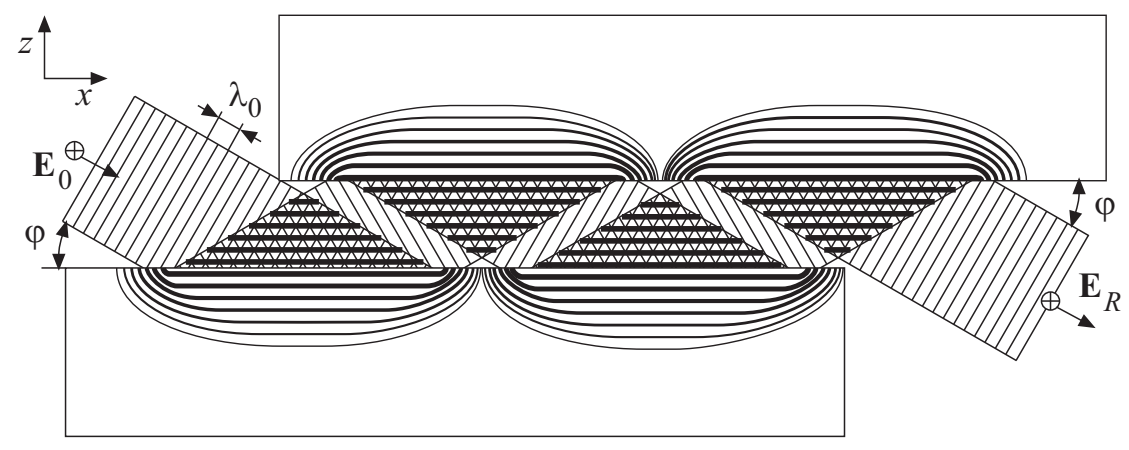

$a$

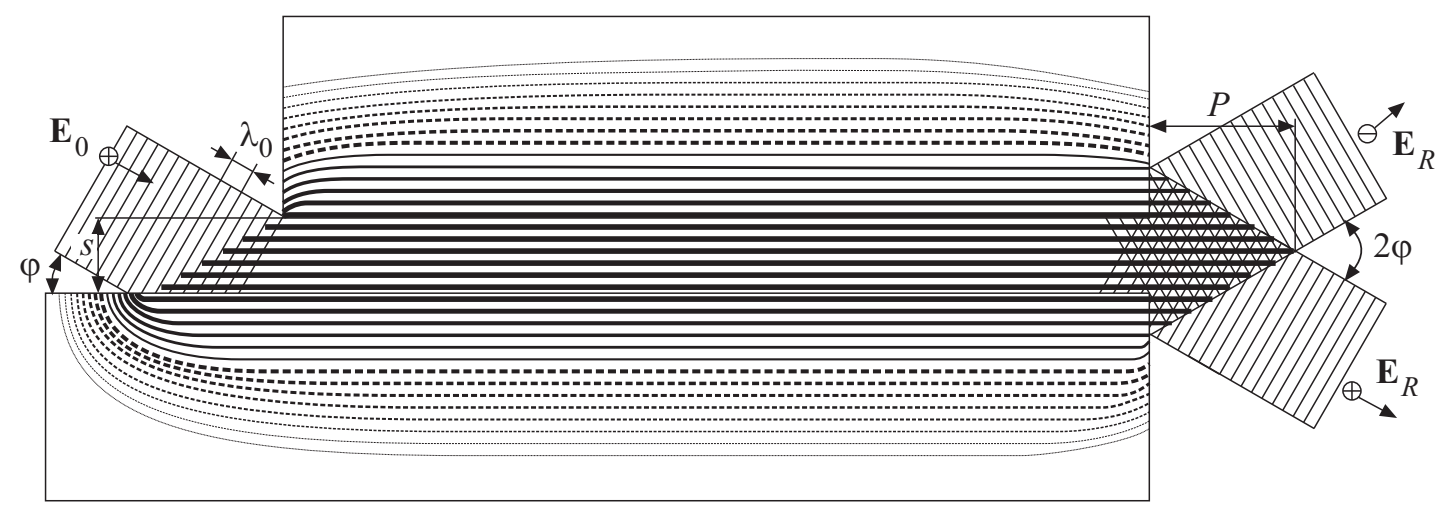

Рис. 2. Модельные представления, характеризующие транспортировку потока квазимонохроматического рентгеновского излучения плоским протяженным щелевым зазором шириной $s>\lambda_{0}^{2} / 2 \Delta \lambda$ в соответствии с механизмом многократного ПВО $(a)$ и плоским протяженным щелевым зазором шириной $s<\lambda_{0}^{2} / 2 \Delta \lambda$ в соответствии с механизмом волноводно-резонансного распространения радиации $(b) . P=\lambda_{0}^{3} / 8 \Delta \lambda^{2}-$ выпучивание однородного интерференционного поля стоячей рентгеновской волны из щелевого зазора в условиях волноводно-резонансного распространения потока.

поверхностей рефлекторов интенсивность этого потока понижается лишь на несколько процентов при длине ПРВР 75-150 mm.

Способность волноводно-резонансных структур захватывать поток квазимонохроматического рентгеновского излучения в свои щелевые зазоры ограничена ПВО этого излучения на поверхности материала рефлектора. Поэтому ПРВР способен захватывать это излучение в угловом интервале, равном двойному критическому углу ПВО для материала рефлектора $\theta_{c}$. Для наиболее часто используемых излучений этот интервал колеблется от $0.1^{\circ}$ до $0.5^{\circ}$. Таким образом, ПРВР захватывает радиационный поток в угловом интервале, не превышающем удвоенного значения $\theta_{c}$, транспортирует его своим щелевым зазором почти без ослабления и формирует на выходе пучок рентгеновских лучей наноразмерной ширины, характеризующийся наличием угловой расходимости, соответствующей углу захвата излучения. При этом важно отметить еще одну особенность рентгеновских пучков, формируемых ПРВР. Эта особенность связана с повышенной радиационной плотностью формируемого потока в сравнении со значением этого параметра в рентгеновских пучках, формируемых потокообразующими структурами [38]. Увеличение радиационной плотности, обычно составляющее 3-4 порядка, является результатом существенного различия в ширине щелевого зазора ПРВР и фокуса источника рентгеновского излучения. В то же время наличие угловой расходимости формируемого на выходе волновода-резонатора радиационного потока нивелирует его важнейшие преимущества - наноразмерность и повышенную радиационную плотность уже на расстоянии нескольких сантиметров от выходного среза ПРВР. Поэтому одной из важнейших практических задач повышения эффективности волноводно-резонансных формирователей потоков рентгеновских лучей явились поиски конструктивных решений, позволяющих уменьшить угловую расходимость формируемого потока в сравнении с углом захвата радиации при сохранении его интегральной интенсивности. На рис. 4 представлена конструкция простейшего ПРВР, а также предложенные нами примеры конструкций волноводно-резонансных устройств, позволяющих понизить угловую расходимость формируемого потока при сохранении его интегральной интенсивности. Наиболее очевидным способом вдвое понизить угловую расходимость выходного потока является конструкция ПРВР на базе использования рефлекторов разной длины (рис. $4, b$ ). В этом случае можно ожидать существенное изменение пространственного распределения интенсивности в потоке рентгеновских лучей от формы, близкой 


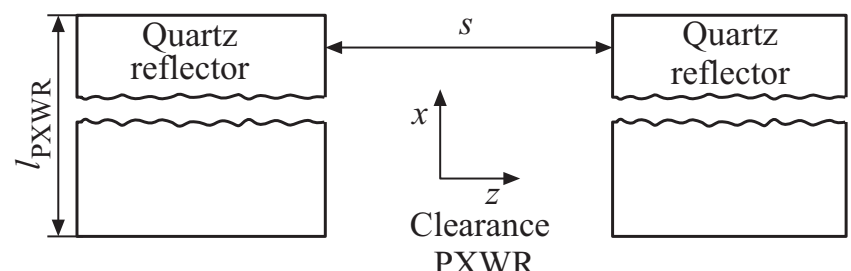

$J(\theta ; z)=I(\theta ; z) \exp (|z| / \Delta z) \quad|z|>s / 2$
$I(\theta ; z)=\left|\mathbf{E}_{0}\right|^{2}\left[1+R^{*}+2 \sqrt{R^{*}} \cos (\psi+2 \pi z / D)\right] \quad|z|<s / 2$

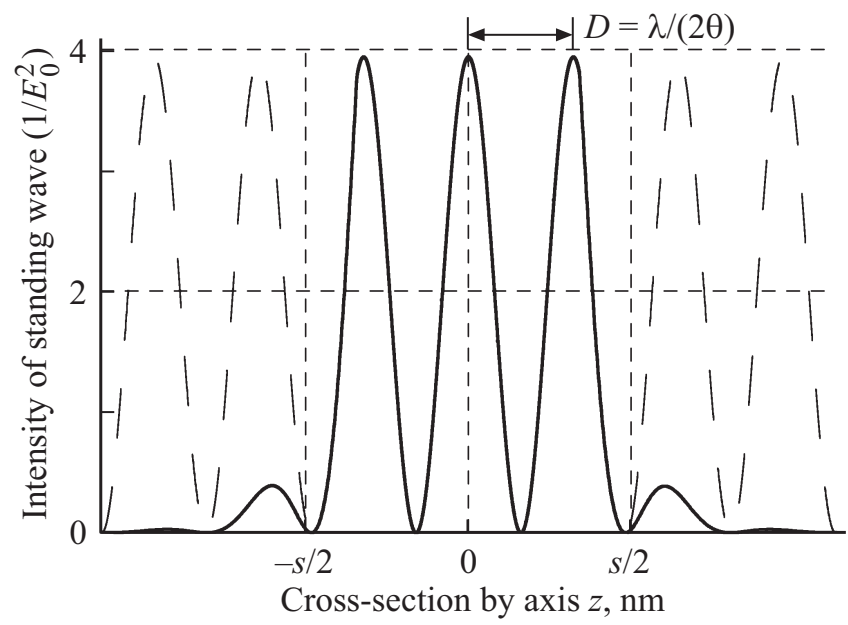

Рис. 3. Функция распределения интенсивности интерференционного поля стоячей рентгеновской волны в плоском протяженном щелевом зазоре в условиях транспортировки потока квазимонохроматического излучения в соответствии с механизмом его волноводно-резонансного распространения.

к гауссовой, к полугауссову распределению. Экспериментальные данные, показанные на рис. 5, подтвердили эти ожидания.

Еще одной конструкцией, которая давала основания ожидать решение поставленной задачи, была волноводно-резонансная структура с градиентными пленочными покрытиями на кварцевых полированных рефлекторах (рис. 4,c). Предполагалось получить пленочные покрытия на основании аморфного твердого раствора $\mathrm{HfO}_{2}-\mathrm{Al}_{2} \mathrm{O}_{3}$ толщиной несколько десятков нанометров, в которых состав непрерывно менялся от практически стехиометрической окиси гафния с плотностью $9.7 \mathrm{~g} / \mathrm{cm}^{3}$ до $\mathrm{Al}_{2} \mathrm{O}_{3}$ с плотностью $3.9 \mathrm{~g} / \mathrm{cm}^{3}$. Плоский рентгеновский волновод-резонатор, построенный на базе таких рефлекторов, позволил бы вдвое уменьшить угловую расходимость формируемого потока без изменения пространственного распределения интенсивности. К сожалению, вследствие технических трудностей осуществить построение данной конструкции пока не удалось.

Конструкция специфического ПРВР, показанная на рис. $4, d$, представляет собой весьма интересную находку. Эта конструкция состоит из двух последовательно установленных и взаимно сьюстированных волноводоврезонаторов простейшего дизайна (рис. $4, a)$ с просветом между ними $\Delta w$, размер которого соответствует следующему соотношению [12]:

$$
\frac{\lambda_{0}}{2 \theta_{c}^{2}} \leq \Delta w \leq \frac{\lambda_{0}^{3}}{8 \Delta \lambda^{2}}
$$

Границы этого интервала для волноводно-резонансной структуры, названной составным плоским рентгеновским волноводом-резонатором (СПРВР), сформированной набором кварцевых рефлекторов, в условиях транспортировки потока $\mathrm{MoK}_{\alpha}$ имеют значения 9-490 $\mu \mathrm{m}$ (для $\mathrm{Cu} K_{\alpha}-5-600 \mu \mathrm{m}$ ). Исследования функционирования этого устройства показали, что он захватывает излучение в том же угловом интервале, что и ПРВР простейшей конструкции, а на его выходе (т.е. на выходе второго ПРВР, образующего СПРВР) формируется поток с меньшей угловой расходимостью в сравнении с углом захвата излучения первым ПРВР при сохранении интегральной интенсивности и форме пространственного распределения интенсивности рентгеновского излучения, близкой к гауссовой. Это наглядно проиллюстрировано рис. $5, b$. Более того, исследования показали, что варьирование просвета $\Delta w$ между последовательно установленными волноводамирезонаторами простейшей конструкции влияет на степень угловой расходимости формируемого рентгеновского потока. К сожалению, сложность взаимной юстировки ПРВР с наноразмерными протяженными щелевыми зазорами и выявленный в результате исследований факт влияния на формирование выходного потока формы и качества обработки торцов рефлекторов, составляющих ПРВР [39], пока не позволили построить неискаженную зависимость степени снижения угловой расходимости сформированного потока от величины просвета $\Delta w$. Однако полученные данные позволили утверждать, что при выполнении соотношения (14) происходит снижение угловой расходимости формируемого потока рентгеновских лучей в сравнении с углом захвата этого потока при сохранении интегральной интенсивности. В качестве рабочей гипотезы, объясняющей наблюдаемые экспериментальные результаты, было сделано предположение, что имеет место эффект частичного углового туннелирования потока рентгеновских лучей в пространстве просвета $\Delta w$ между волноводамирезонаторами [12].

\section{Предполагаемая модель функционирования СПРВР}

При расстоянии между последовательно установленными ПРВР, превышающем значение $\lambda_{0}^{3} / 8 \Delta \lambda^{2}$ (рис. $6, a$ ), такая структура формирует малорасходящийся рентгеновский пучок. Однако его интегральная интенсивность оказывается существенно меньшей в сравнении с интенсивностью потока, сформированного на выходе первого волновода-резонатора. В этом случае однородные интерференционные поля стоячей рентгеновской волны, 


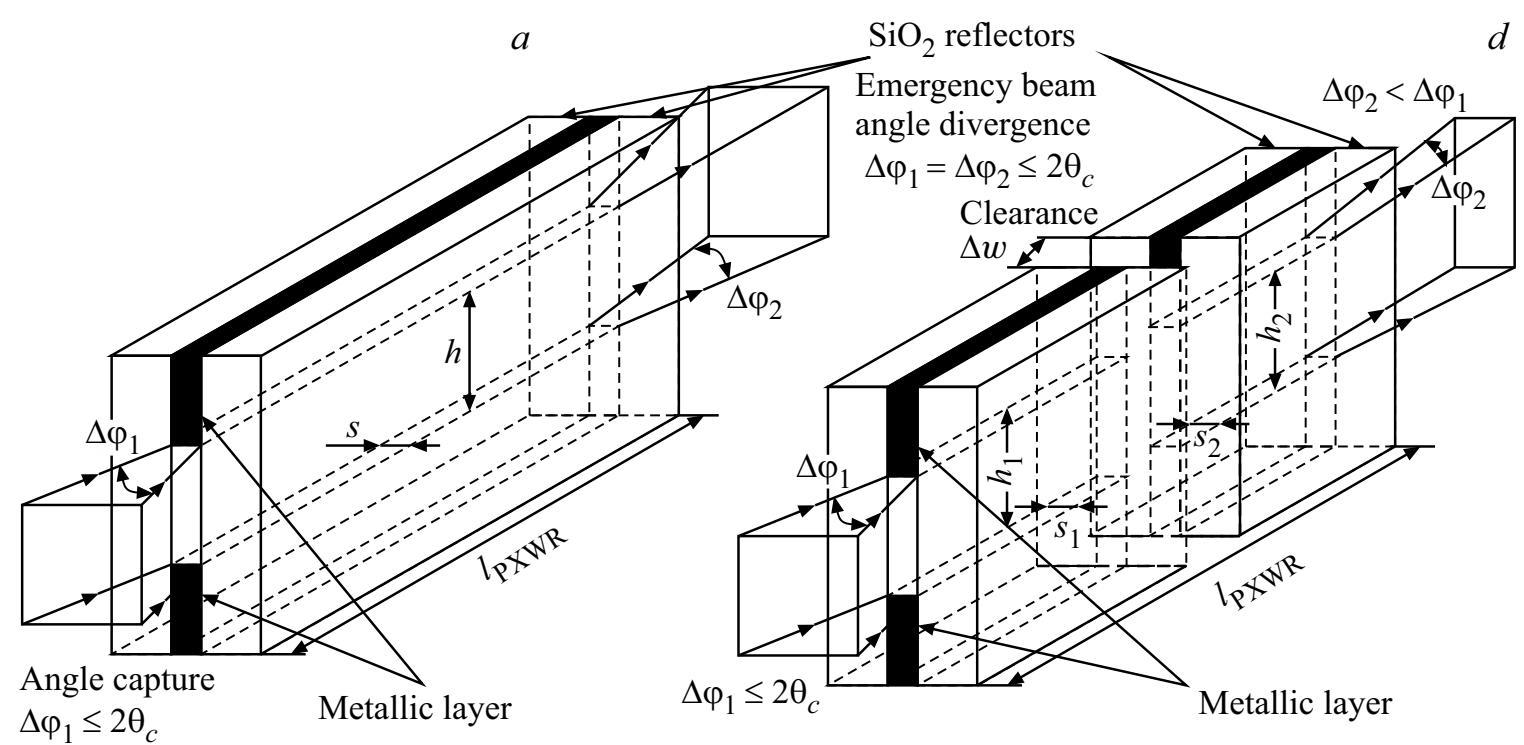

$c$

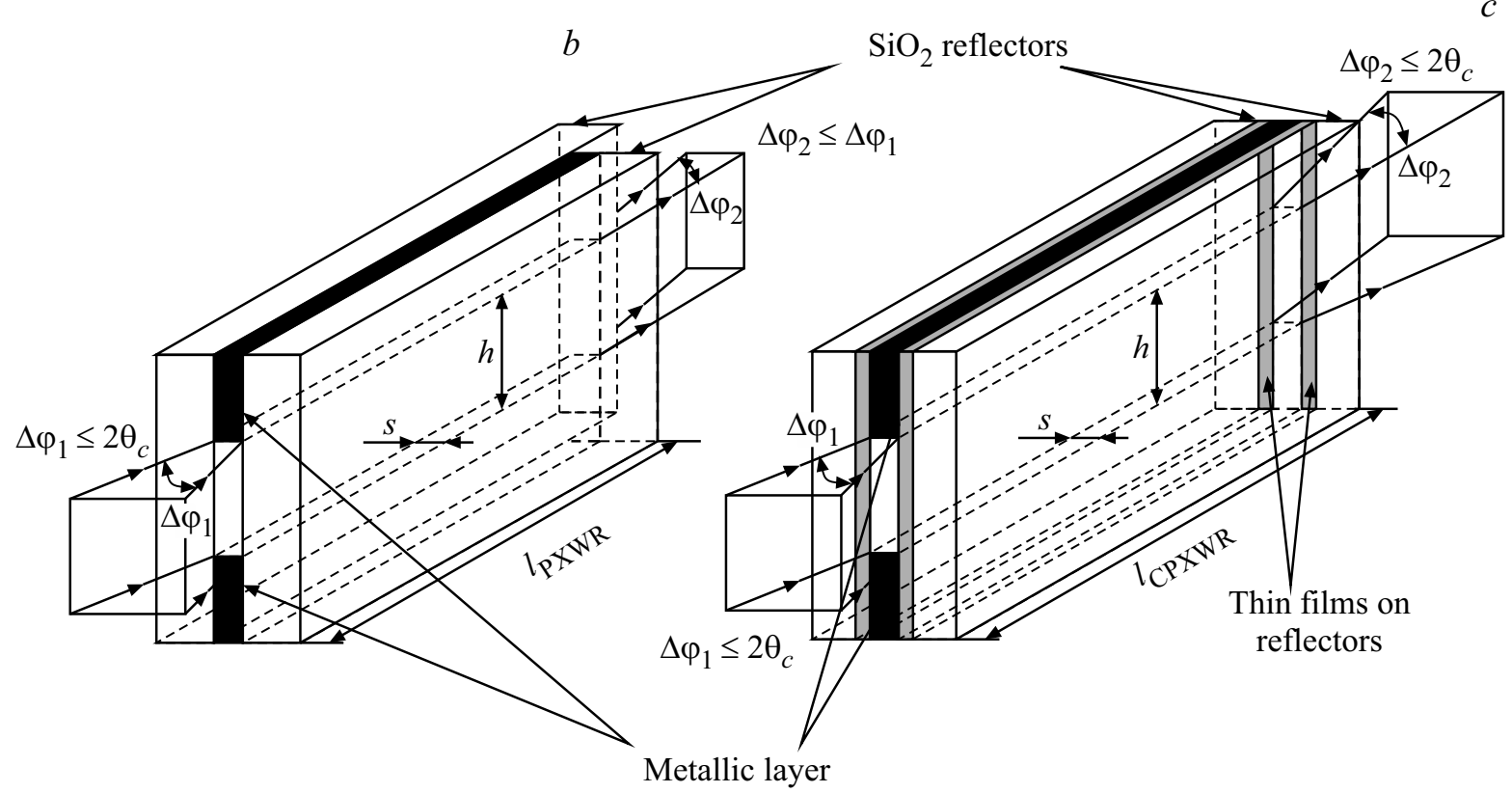

Рис. 4. Схематическое устройство волноводов-резонаторов потоков квазимонохроматического рентгеновского излучения различного дизайна: $a-$ ПРВР простейшей конструкции с кварцевыми рефлекторами; $b-$ ПРВР, сформированный кварцевыми рефлекторами различной длины; $c$ - волноводно-резонансная структура с тонкопленочным градиентным покрытием на кварцевых плоских полированных пластинах; $d-$ составной волновод-резонатор.

возбужденные в первом и втором ПРВР, оказываются независимыми, и интенсивность рентгеновского потока, формируемого на выходе второго волновода-резонатора, определяется только геометрическим фактором. В то же время, если расстояние между последовательно установленными ПРВР станет меньше указанного выше значения, ситуация на выходе второго волновода-резонатора кардинально изменится (рис. $6, b$ ). Возникающее изменение связано с тем, что однородное интерференционное поле стоячей рентгеновской волны, возникающее в щелевом зазоре первого ПРВР, характеризуется некоторым „выпячиванием“, размер которого определяется углом захвата парциального рентгеновского потока (ве- личина $P$ (рис. 2) соответствует захвату излучения с минимально возможным угловым значением). В этом случае в начальный период времени второй волноводрезонатор захватит в свой щелевой зазор парциальный рентгеновский поток, интенсивность которого будет определяться чисто геометрическими параметрами. Этот поток сформирует интерференционное поле стоячей рентгеновской волны, соответствующее интенсивности этого парциального потока. Однако вследствие того, что поле первого ПРВР оказывается проникающим в щелевой зазор второго волновода-резонатора, интерференционные поля начинают взаимодействовать, достигая некоторого нового состояния. Их взаимодей- 

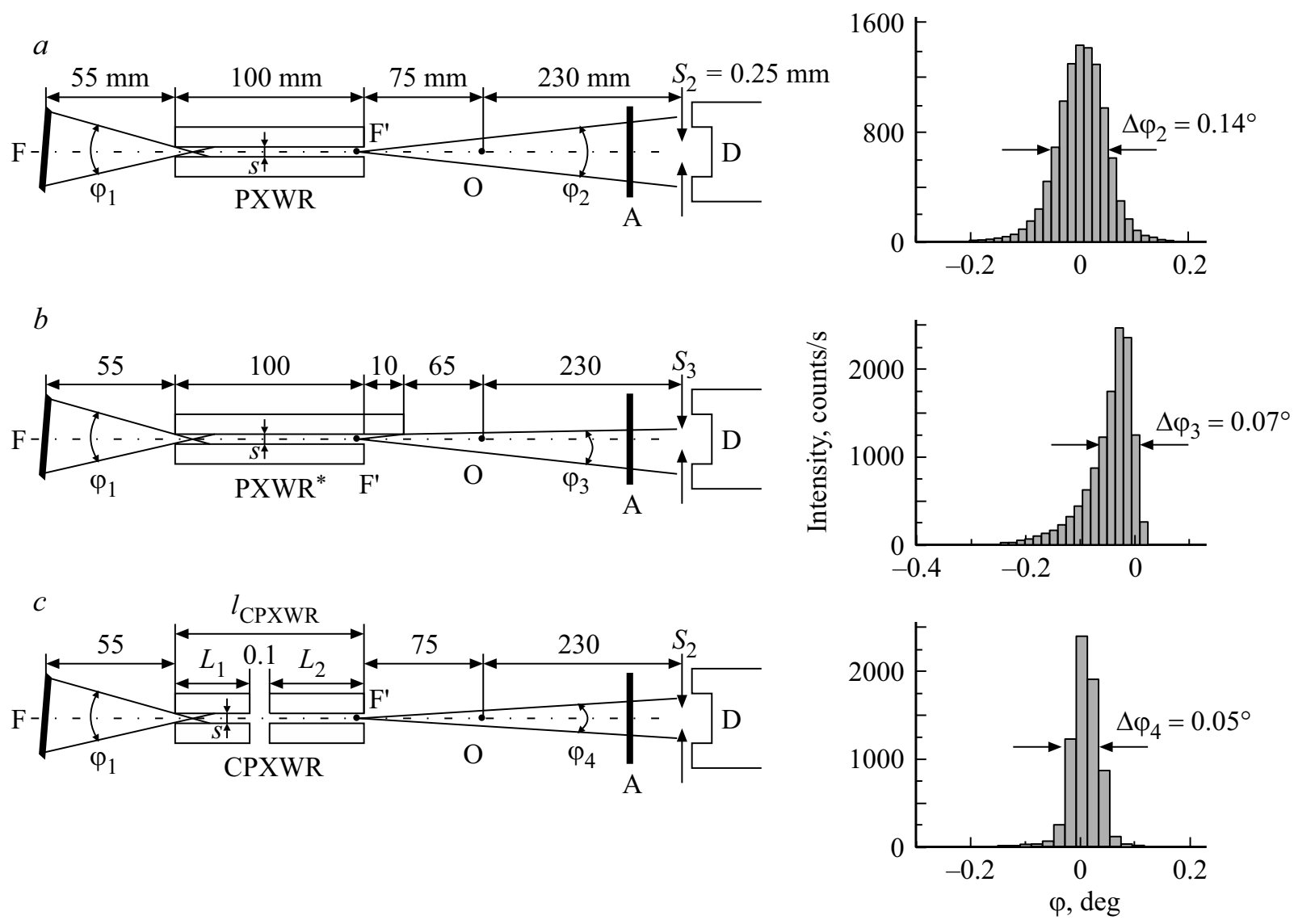

Рис. 5. Схемы формирования потоков квазимонохроматического рентгеновского излучения с помощью кварцевого ПРВР простейшей конструкции $(a)$, волновода-резонатора, построенного на базе разноразмерных рефлекторов $(b)$ и СПРВР $(c)$, а также пространственное распределение интенсивности излучения $\mathrm{Cu} K_{\alpha}$ в потоках, сформированных этими устройствами. Распределения зарегистрированы в условиях работы источника БСВ-24 $(\mathrm{Cu}) U=20 \mathrm{keV}, J=10 \mathrm{~mA}$. Коэффициент поглощения фильтра А $K=50$. Ширина щелей волноводно-резонансных устройств $s=40 \mathrm{~nm}$.

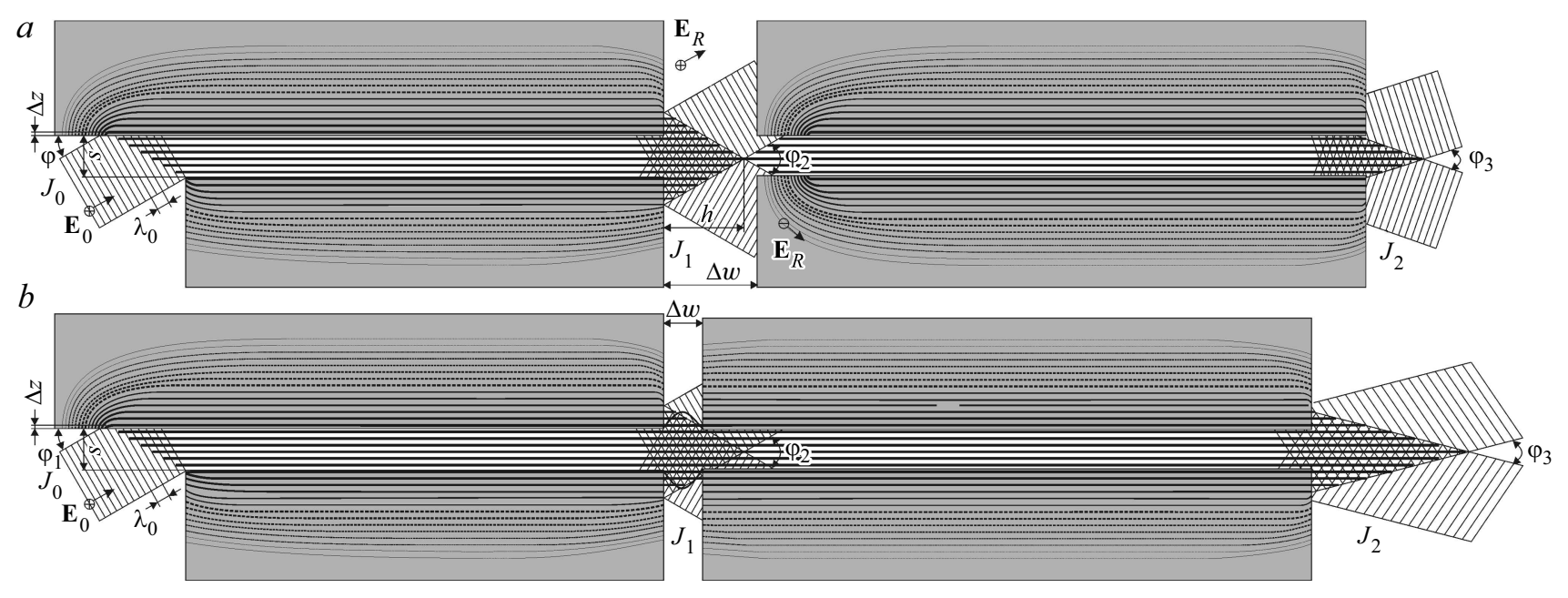

Рис. 6. Схемы формирования потока квазимонохроматического рентгеновского излучения парой установленных друг за другом и взаимно съюстированных ПРВР с шириной щелевых зазоров $s=40 \mathrm{~nm}$ : $(a)$ с расстоянием между ПРВР $\Delta w>\lambda_{0}^{3} / 8 \Delta \lambda$, не допускающим частичного углового туннелирования потока квазимонохроматического рентгеновского излучения; $(b)$ с расстоянием между ПРВР $\Delta w<\lambda_{0}^{3} / 8 \Delta \lambda^{2}$, реализующим радиационное угловое туннелирование. 
ствие приводит к тому, что часть рентгеновского потока, сформированного на выходе первого ПРВР и не способного в соответствии с геометрическим фактором попасть в щелевой зазор второго волновода-резонатора, меняет направление распространения и туннелирует в щелевой зазор второго ПРВР. Эффект туннелирования приводит к росту интенсивности потока в щелевом зазоре второго ПРВР и соответственно повышению интенсивности интерференционного поля стоячей рентгеновской волны в его щелевом зазоре. Это, в свою очередь вызывает изменение параметра взаимодействия интерференционных полей первого и второго ПРВР и ведет к постепенному установлению стационарного режима транспортировки рентгеновского потока. При этом экспериментальные исследования показали (например, данные рис. $5, c)$, что достигаемый стационарный режим характеризуется сохранением интегральной интенсивности квазимонохроматического рентгеновского потока и снижением угловой расходимости этого потока в сравнении с углом захвата радиации первым волноводом-резонатором. Таким образом, предлагаемая умозрительная модель дает логическое объяснение экспериментально наблюдаемому эффекту, но при этом абстрагируется от важнейшего постулата теоремы Лиувилля о неизменности объема фазового пространства, описывающего любой статистический континуум [40]. Экспериментальные данные показывают, что в результате прохождения через составной волновод-резонатор поток рентгеновского квазимонохроматического излучения сохраняет свою интегральную интенсивность, но при этом характеризуется пониженной угловой расходимостью. В то же время совершенно ясно, что характеристика рентгеновского потока, формируемого на выходе СПРВР, не ограничивается указанными двумя параметрами, и для уточнения предлагаемой модели функционирования СПРВР необходимо выявить тот параметр потока, который в соответствии с теоремой Лиувилля должен претерпевать предполагаемые изменения. Анализ возможных параметрических изменений показал, что наиболее вероятным фактором, сопровождающим уменьшение угловой расходимости квазимонохроматического рентгеновского потока, формируемого на выходе СПРВР, является ухудшение его монохроматичности, т.е. увеличение $\Delta \lambda$ и соответственно снижение длины когерентности этого потока. Данное предположение детально обсуждалось в рамках доклада, сделанного авторами на 25 Международном симпозиуме „Наноструктуры: физика и технология“ [41], и было проверено экспериментально.

На рис. 7 представлены фрагменты дифрактограмм монокристаллического кремния, срезанного перпендикулярно плоскости (111), в области рефлекса (111), полученные с использованием источника излучения БСВ-24 $(\mathrm{Cu})$ в режиме $U=20 \mathrm{keV}, J=10 \mathrm{~mA}$ в условиях формирования первичного потока излучения с помощью ПРВР простейшей конструкции (a) и составного волновода-резонатора $(b)$. Дифрактограммы были зарегистрированы в режиме высокого углового разрешения на гониометре HZG-4 (минимальный шаг сканирования $\delta \varphi=0.0005^{\circ}$ ) в геометрии БрегаБрентано с радиусом измерений $R=235 \mathrm{~mm}$ и шириной детекторной щели $S_{d}=0.1 \mathrm{~mm}$. Для измерений были использованы волноводно-резонансные устройства с плоскими кварцевыми щелевыми зазорами $s=40 \mathrm{~nm}$ и длиной $l=100 \mathrm{~mm}$. Расстояние между последовательно установленными волноводами-резонаторами в СПРВР составляло $\Delta w=0.1 \mathrm{~mm}$. Интегральные и угловые характеристики потоков на выходе ПРВР и СПРВР соответствуют данным, приведенным на рис. 5. Сравнительные дифрактометрические данные, представленные на рис. 7, указывают на небольшое увеличение ширины рефлекса при использовании в качестве формирователя исходного рентгеновского потока составного волноводарезонатора. Наблюдаемое уширение является прямым подтверждением предварительно сделанного предположения о снижении степени монохроматичности потока излучения после его транспортировки СПРВР, хотя для количественной оценки этого факта представленных измерений недостаточно. Однако полученные качественные данные позволяют утверждать, что в соответствии с требованиями теоремы Лиувилля снижение угловой расходимости потока квазимонохроматического рентгеновского излучения компенсируется ухудшением его монохроматичности. Несмотря на то что эти два параметра не являются коммутирующими, можно попытаться найти аналитическую связь между их взаимными изменениями.

Энергия и импульс одиночного фотона в потоке электромагнитного излучения $E_{\rho}$ и $P_{\rho}$ определяются [34] выражениями

$$
\begin{gathered}
E_{\rho}=\hbar \omega=h c / \lambda_{0}=P_{\rho} c, \\
P_{\rho}=E_{\rho} / c=h / \lambda_{0},
\end{gathered}
$$

где $h=2 \pi \hbar$ является постоянной Планка, $\omega=2 \pi f$ и $\lambda_{0}$ представляют собой угловую частоту и среднюю длину волны излучения, $c$ - скорость света в вакууме. Дифференцируя формулы (15) и (16), можно получить связь между изменениями энергии и импульса отдельно взятого фотона с вариацией длины волны излучения:

$$
\begin{gathered}
\Delta E_{\rho}=-\frac{h c}{\lambda_{0}^{2}} \Delta \lambda=\frac{P_{\rho}^{2} c}{h} \Delta \lambda, \\
\Delta P_{\rho}=-\frac{h}{\lambda_{0}^{2}} \Delta \lambda=-\frac{P_{\rho}}{\lambda_{0}} \Delta \lambda=\frac{P_{\rho}^{2}}{h} \Delta \lambda .
\end{gathered}
$$

Как было описано ранее, часть потока квазимонохроматического рентгеновского излучения, сформированного первым ПРВР, составляющим составной волноводрезонатор, попадает во второй ПРВР и инициирует в нем однородное интерференционное поле стоячей 


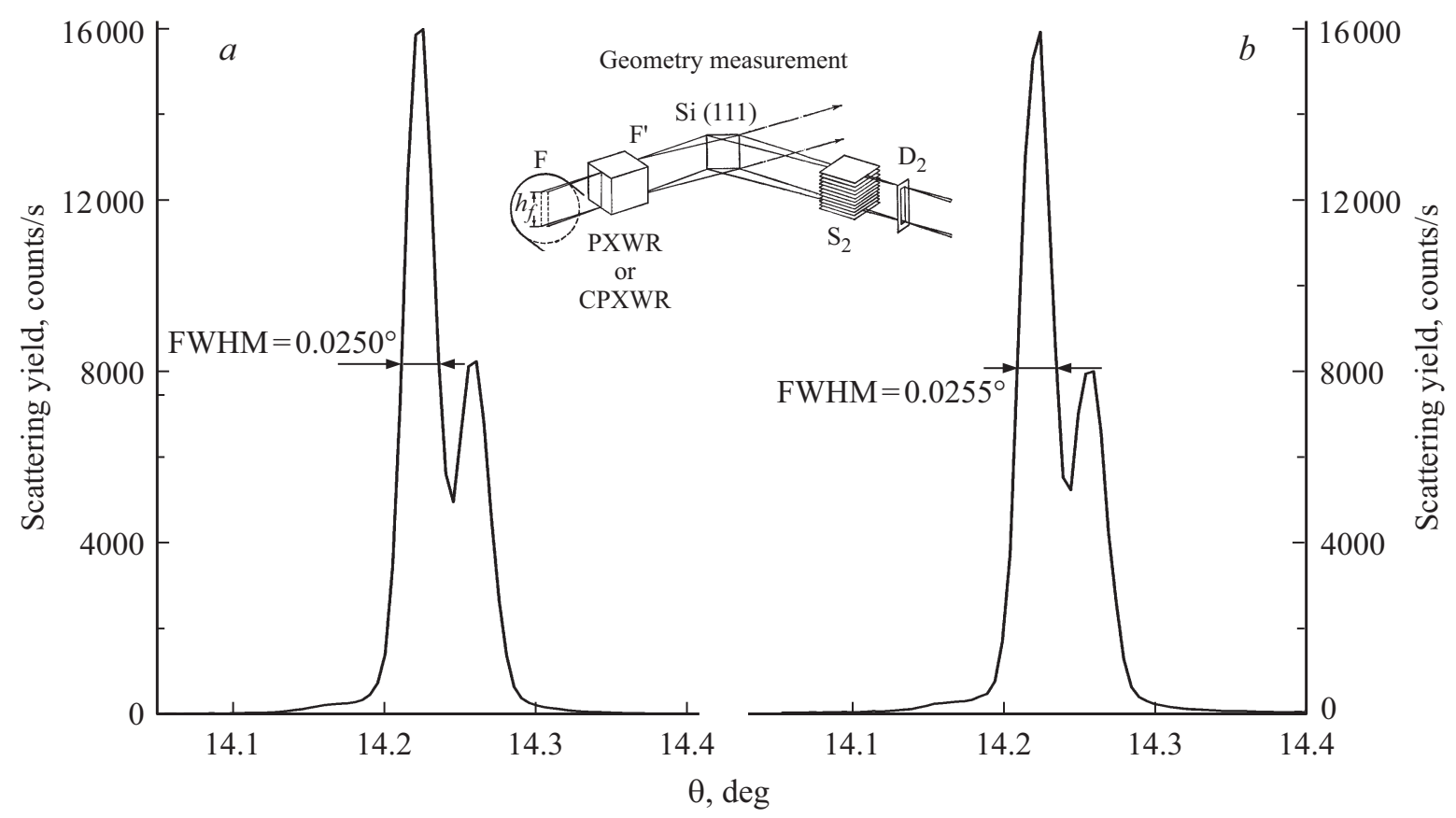

Рис. 7. Фрагменты дифрактограммы рефлекса (111) монокристалла кремния, срезанного параллельно плоскости (111), полученные в условиях применения в качестве формирователя исходного рентгеновского пучка волновод-резонатор простейшей конструкции с шириной щелевого зазора $s=40 \mathrm{~nm}(a)$, и СПРВР с аналогичной шириной щелевого зазора $(b)$. Фрагменты дифрактограмм зарегистрированы с использованием источника излучения БСВ-24 (Cu) в режиме $U=20 \mathrm{keV}, J=10 \mathrm{~mA}$ на цифровом гониометре HZG-4, радиус $R=235 \mathrm{~mm}$, шаг измерений $\Delta \theta=0.005^{\circ}$.

рентгеновской волны. В результате взаимодействия интерференционных полей первого и второго ПРВР устанавливается стационарный режим транспортировки излучения, причем часть потока, сформированного первым ПРВР, не способная попасть во второй ПРВР по геометрическим соображениям, туннелирует в промежутке $\Delta w$ во второй ПРВР. Предположим, что фотоны этой части рентгеновского потока, сформированного первым ПРВР, испытывают угловое туннелирование и характеризуются вектором импульса $\mathbf{P}_{1}$, равным $\mathbf{P}_{p}$, направленным под углом $\varphi_{1}$ к оси СПРВР. После прохождения второго ПРВР эта часть потока будет характеризоваться вектором $\mathbf{P}_{2}$, равным $\mathbf{P}_{\rho}+\delta \mathbf{P}_{\rho}$, направленным по отношению к оси составного волновода-резонатора под углом $\varphi_{2}$ (рис. 8). Экспериментальные данные показывают, что $\varphi_{1}>\varphi_{2}$, и изменение угловой апертуры для этой части потока будет представлено как:

$$
\Delta \varphi=\varphi_{1}-\varphi_{2} .
$$

Поскольку рентгеновские волноводно-резонансные структуры функционируют в рамках явления ПВО потоков рентгеновского излучения на материальной поверхности, значения углов $\varphi_{1}$ и $\varphi_{2}$, а также их разности являются малыми величинами. Поэтому для тангенсов этих углов возможно использование разложения в ряд [42], игнорируя члены старших порядков:

$$
\Delta \varphi=\left(\varphi_{1}-\varphi_{2}\right) \approx \frac{P_{y 1}}{P_{x 1}}-\frac{P_{y 2}}{P_{x 2}} .
$$
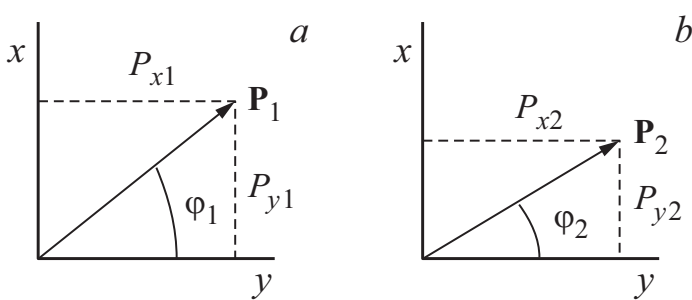

Рис. 8. Схематическое представление импульса фотона до $(a)$ и после $(b)$ туннелирования. $P_{1}$ соответствует исходному значению импульса $P_{p}, P_{2}$ соответствует новым значениям $P_{p}+\delta P_{p}$ и $P_{p}-\delta P_{p}$.

При вариации значений малых углов координата $x$ вектора импульса фотонов, претерпевших эффект туннелирования, меняется незначительно, поскольку она описывается функцией косинуса этих углов. Поэтому выражение (20) может быть преобразовано к виду

$$
\Delta \varphi \approx \frac{P_{y 1}-P_{y 2}}{P_{x 1}} \approx \frac{\Delta P_{y}}{P_{\rho}} .
$$

С другой стороны, скалярное значение импульса этих фотонов может быть представлено в общепризнанном формате:

$$
P_{p}^{2}=P_{x}^{2}+P_{y}^{2} .
$$

В результате дифференцирования этого выражения получаем соотношение между общим изменением импуль- 
са и изменением его проекций:

$$
P_{p} \frac{\Delta P}{\Delta \varphi}=P_{x} \frac{\Delta P_{x}}{\Delta \varphi}+P_{y} \frac{\Delta P_{y}}{\Delta \varphi} .
$$

Поскольку вариация составляющей импульса по координате $x$ в результате туннелирования фотонов значительно меньше изменения его составляющей по второй координате, в первом приближении выражение (21) может быть представлено в виде

$$
\Delta P_{p}=P_{y} \Delta \varphi=\Delta \varphi P_{p} \sin \varphi_{1} \approx P \varphi_{1} \Delta \varphi
$$

Сопоставляя выражения (18) и (24), может быть получена реальная связь между снижением угловой расходимости части потока квазимонохроматического рентгеновского излучения $\Delta \varphi$ и ухудшением монохроматичности этой части потока на величину $\delta \lambda$ :

$$
\delta \lambda=\varphi_{1} \lambda_{0} \Delta \varphi
$$

Совершенно понятно, что данное выражение справедливо лишь для малых углов, характерных для наблюдения явления ПВО потоков рентгеновских лучей на материальном интерфейсе. Кроме того, необходимо помнить, что ухудшение монохроматичности излучения характерно лишь для той части потока, сформированного составным волноводом-резонатором, которое претерпело туннелирование в межволноводно-резонансном промежутке $\Delta w$.

В любом случае представляется полезным оценить изменение степени монохроматичности потока рентгеновского квазимонохроматического излучения в результате его туннелирования в промежутке $\Delta w$. Наши экспериментальные исследования показали (рис. 5,c), что тестированный нами СПРВР снизил угловую расходимость в формируемом потоке в сравнении с углом захвата почти в три раза. Для оценок можно представить идеальный случай формирования на выходе СПРВР коллинеарного рентгеновского потока, что будет соответствовать максимально возможной величине $\Delta \varphi$. Следовательно, максимальное значение снижения степени монохроматичности вследствие туннелирования потока будет характеризоваться величиной

$$
\delta \lambda_{\max }=2 \varphi_{1} \lambda_{0}
$$

Для излучения $\mathrm{Cu} K_{\alpha}\left(\lambda_{0}=0.1541 \mathrm{~nm}\right)$ оцениваемая нами величина $\delta \lambda_{\max }$ составляет $4.0 \cdot 10^{-4} \mathrm{~nm}$. Для сравнения можно указать, что значение $\Delta \lambda$ для генерируемого лабораторным стандартным источником характеристического рентгеновского излучения $\mathrm{Cu} K_{\alpha}$ составляет $0.44 \cdot 10^{-4} \mathrm{~nm}[43]$, т. е. туннелирование излучения в промежутке $\Delta w$ составного волновода-резонатора способно снизить степень монохроматичности излучения, генерируемого лабораторными источниками рентгеновского излучения, практически на порядок. В эксперименте, представленном на рис. 5, $c$, протуннелировавшая часть рентгеновского потока характеризуется троекратным ухудшением степени монохроматизации, что реально привело к некоторому уширению дифракционной картины. Таким образом, с практической точки зрения, использование составных волноводно-резонансных структур для формирования потоков возбуждения для рентгенофлуоресцентного анализа представляется вполне оправданным, в то время как применение таких потоков в дифрактометрии может приводить к появлению определенных трудностей.

Как уже указывалось выше, наши экспериментальные исследования показали, что варьирование ширины промежутка $\Delta w$ между волноводами-резонаторами в СПРВР влияет на расходимость формируемого потока $\Delta \varphi$. Однако из этих же измерений стало ясно, что на реальную расходимость потока влияет еще и целый ряд технологических параметров. Поэтому получение количественного соотношения между величинами $\Delta w, \Delta \varphi$ и $\Delta \lambda$ представляется в настоящее время проблематичным. В то же время, предложенная модель углового туннелирования части потока рентгеновского излучения, увязывающая между собой уменьшение угловой расходимости потока, формируемого СПРВР, и снижение степени монохроматичности части потока, претерпевшего угловое туннелирование, представляется вПолне логичной и обоснованной. При этом хотелось бы обратить внимание на важное следствие предложенной модели. Дело в том, что средняя длина волны той части сформированного потока, которая испытала туннелирование, сохранила свою величину как $\lambda_{0}$. В то же время в этой части потока появились фотоны, которые изменили свою длину волны на величины $\pm \delta \lambda / 2$. Следовательно, в этой части потока появились фотоны с энергией и импульсом, возросшими и уменьшенными в результате туннелирования на значения

$$
\begin{gathered}
\delta E= \pm \frac{h c}{\lambda_{0}} \varphi_{1} \Delta \varphi, \\
\delta P= \pm P_{p} \varphi_{1} \Delta \varphi .
\end{gathered}
$$

Конечно, изменения $\delta E$ и $\delta P$, связанные с туннелированием части рентгеновского потока, будут наложены на наличие естественного энергетического и импульсного разброса в потоке характеристических излучений, генерируемых лабораторными источниками, определяемого исходной величиной $\Delta \lambda$ и соответственно $\Delta E$ и $\Delta P$. Само значение $\Delta \lambda$ и, следовательно, $\Delta E$ в исходном потоке может быть значительно уменьшено путем использования качественных монохроматоров. В этом случае, вероятно, удастся выделить парциальные потоки с измененной в результате туннелирования энергией. Сам по себе факт появления фотонов с уменьшенной и увеличенной энергиями некоторым образом напоминает комбинационное рассеяние света с появлением в результирующем спектре стоксовской и антистоксовской компонент. Однако более детальное исследование явления частичного углового туннелирования потока квазимонохроматического рентгеновского излучения в настоящее время нуждается 
в разработке более совершенной технологии приготовления рефлекторов ПВО рентгеновского излучения, используемых для формирования плоских протяженных волноводно-резонансных структур, используемых для сборки СПРВР.

\section{Заключение}

В работе дана краткая характеристика волноводнорезонансного распространения потоков квазимонохроматического рентгеновского излучения и устройств, функционирующих в рамках этого явления. Особое внимание уделено обсуждению особенности формирования пучков рентгеновского излучения СПРВР, связанной с явлением частичного углового туннелирования потока излучения в промежутке между последовательно установленными ПРВР, составляющими СПРВР. Предложена самосогласованная модель, описывающая это явление, которая позволила оценить связь между наблюдаемым экспериментально уменьшением расходимости радиационного потока на выходе составного волновода-резонатора и ожидаемым ухудшением монохроматичности излучения в этом потоке. Констатировано, что дальнейший прогресс в улучшении параметров рентгеновских пучков, формируемых СПРВР, связан с совершенствованием технологии изготовления элементов, составляющих составные волноводно-резонансные структуры.

Работа выполнена при частичной финансовой поддержке РФФИ, грант № 15-08-02618. Публикация подготовлена при поддержке Программы РУДН „5-100“.

\section{Список литературы}

[1] Schaefer C. Einfunhrung in die theoretische physik, Electrodynamik und optic. Berlin: Walter de gruyter, 1932. Перевод: Шефбер К. Теоретическая физика. Т. 3. Ч. 2. Оптика. М.: ГОНТИ, 1938. 536 с.

[2] Ditchborn R.W. F.R.S. Light. London: Blackie \& Son Limited, 1963. Перевод: Дитчборн Р. Физическая оптика. М.: Наука, 1965. $631 \mathrm{c}$.

[3] Harrik N.J. Internal reflection spectroscopy. N.Y.: Wiley, 1967. Перевод: Харрик Н. Спектроскопия внутреннего отражения. М.: Мир, 1970. 335 с.

[4] Milosevic M. Internal reflection and ATR spectroscopy. New Jersey: Wiley, 2012. $239 \mathrm{p}$.

[5] Лебедева В.В. Техника оптической спектроскопии. М.: МГУ, 1977. $383 \mathrm{c}$.

[6] Hecht E. Optics. 4 Ed. San Francisco: Addison Wesley, 2002. $698 \mathrm{p}$.

[7] James P.W. The optical principles of the diffraction of Xrays. The crystalline state. V. 2. London. 1948. Перевод: Джсеймс Р. Оптические принципы дифракции рентгеновских лучей. М.: ИИЛ, 1950. 572 с.

[8] Алихонов А.И. Оптика рентгеновских лучей. М.: ГТТИ, 1933. $104 \mathrm{c}$
[9] Иверонова В.И., Ревкевич Г.П. Теория рассеяния рентгеновских лучей. М.: МГУ, 1978. 277 с.

[10] Snigirev A., Kohn V., Snigireva I. et al. // Nature. 1996. V. 384. P. 49.

[11] Egorov V.K., Egorov E.V. // X-ray spectrometry. 2004. V. 33. P. 360.

[12] Егоров Е.В., Егоров В.К. // Поверхность (рент, синх., нейтр. исследования). 2005. № 2. С. 64.

[13] Мингазин Т.А., Зеленов В.И., Лейкин В.Н. // ПТЭ. 1981. № 1. C. 229.

[14] Лейкин В.Н., Мингазин Т.А., Зеленов В.И. // ПТЭ. 1981. № 3. C. 208.

[15] Лейкин В.Н., Мингазин Т.А., Зеленов В.И. // ПТЭ. 1984. № 6. C. 33 .

[16] Kumahov M., Komarov F. // Phys. Rep. 1990. V. 191. P. 289.

[17] Zwanenburg M.J., Peters J.F., Bongaerts J.H.H. et al. // Phys. Rev. Lett. 1999. V. 82. P. 1696.

[18] Hirch P.B., Kellar J. // Proc. Phys. Soc. London Scr. 1951. V. 64. P. 369.

[19] Егоров В.К., Зуев А.П., Малюков Б.А. // Известия вузов. Цветная металлургия. 1997. № 5. С. 54.

[20] Egorov V.K., Kondratiev O.S., Zuev A.P. et al. // Adv. X-ray Anal. 2000. V. 43. P. 406.

[21] Егоров В.К., Зуев А.П., Егоров Е.В. // Заводская лаборатория. 2001. Т. 67. С. 3.

[22] Bedzik M., Bommarito G., Schildkraut J. // Phys. Rev. Lett. 1989. V. 69. P. 1376.

[23] Born M., Wolf E. Principles of optics. Oxford: Pergamon Press, 1965. Перевод: Борн М., Воль $ђ$ Э. Основы оптики. М.: Наука, 1973. 719 с.

[24] Вольф Э., Мандель Л. // УФН. 1965. Т. 87. С. 491; 1966. T. 88. C. 347 ; 1966. T. 88. C. 619.

[25] Lanterborn W., Kurz T. Coherent Optics. Berlin: Springer, 1995. $300 \mathrm{p}$.

[26] Mandel L., Wolf E. Optical coherence and quantum optics. Cambridge: Cambridge Univ. Press, 1995. 837 р. Перевод: Мандель Л., Воль $\oint$ Э. Оптическая когерентность и квантовая оптика. М.: Физматлит, 2000. 895 с.

[27] Egorov V.K., Egorov E.V. // X-ray Spectrometry. 2007. V. 36. P. 381.

[28] Compton A.H., Allison S.K. X-ray in theory and experiment. N.Y.: Nostrand Com., 1935. 828 р. Перевод: Комптон A., Алисон С. Рентгеновские лучи, теория и эксперимент. Л.: ОГИЗ ГИТТЛ, 1941. $672 \mathrm{c.}$

[29] Блохин М.А. Физика рентгеновских лучей. М.: ГИТТЛ, 1953. $455 \mathrm{c}$.

[30] Ландау Л.Д., Лифшии, Е.М. Электродинамика сплошных сред. М.: ГИФМЛ, 1959. $532 \mathrm{c.}$

[31] Lekner J. Theory of reflection of electromagnetic and particle waves. Dordrecht: Martinys Nijhoff Publ., 1987. 279 p.

[32] Drude P. Lehrbuch der optics. Leipzig: Verlag, 1912. Пepeвод: Друде П. Оптика. Л.: ГРОЛ, 1935. 470 с.

[33] Parrat L.G. // Phys. Rev. 1954. V. 95. P. 359.

[34] Bohm D. Quantum theory. NY.: Prentice Hall, 1952. Перевод: Бом Д. Квантовая теория. М.: Наука, 1965. 727 с.

[35] Goos F., Hanchen H. // Ann. Phys. 1947. V. 1. P. 333.

[36] Jakiel J., Kontor W. // Eur. Phys. J. D. 2014. 68. 305. P. 1.

[37] Егоров В., Егоров Е. Планарные рентгеновские волноводы-резонаторы. Реализация и перспективы. Saarbrucken: Lamber Acad. Publ., 2017. 388 c.

[38] Быков В.А., Егоров В.К., Егоров Е.В. Патент РФ № 2528561, 2010. 
[39] Егоров Е.В., Егоров В.К. // Тезисы докладов РСНЕ-2009, М.: РНЦ Курчатовский институт, 2009. С. 578.

[40] Френкель Я.И. Статистическая физика. М.: АН СССР, 1948. $760 \mathrm{c}$.

[41] Egorov V.K., Egorov E.V. // Conf. Proc. 25th International Symposium „Nanostructures: physics and technology“, St. Petersburg: Academic Univ. Publ., 2017. P. 264.

[42] Бронштейн И.Н., Семендяев К.А. Справочник по математике для инженеров и учащихся вузов. М.: Наука, 1964. $608 \mathrm{c}$.

[43] Блохин М.А., Швейцер И.Г. Рентгеноспектральный справочник. М.: Наука, 1982. С. 376. 